\title{
How to Divide the Possession of a Football?
}

\author{
Yeon-Koo Che* Terry Hendershott ${ }^{\dagger}$
}

August 31, 2007

\begin{abstract}
In an National Football League overtime, a coin is tossed to determine which team will receive the kick off. In the sudden death format starting on offense has a significantly higher chance of winning. We examine two proposals to improve the ex post fairness: auctions and divide-and-choose. We find the auction to provide a better outcome than the divide-and-choose rule in general when the teams have asymmetric assessments about how the overtime game may unfold. The result has broad implications for resource division when individuals do not have complete information about the objects being divided.

Keywords: Fair Division, Envy-freeness, Auctions, Divide-and-Choose.
\end{abstract}

*Department of Economics, Columbia University; email: yc2271@columbia.edu.

${ }^{\dagger}$ Haas School of Business, UC-Berkeley; email: hender@haas.berkeley.edu. 


\section{Introduction}

In the National Football League (NFL), games ending in a tie are determined by suddendeath overtime where the first team to score wins. Sudden death is an efficient means to decide a game which is violent and exhausting 11 However, the sudden death nature confers a significant advantage on the team who has the first possession. While the outcome of a coin flip to determine first possession is ex ante fair, immediately after the toss it is no longer fair because the winning team has a significant chance of scoring on its first possession. NFL Commissioner Paul Tagliabue has said that "There has been a trend in the last seven or eight seasons that the team winning the toss in overtime wins the game. That advantage of receiving the ball first is becoming unbalanced." (New York Times, 2003a) ${ }^{2}$

Economic theory suggests natural solutions to this problem. One proposal would have the coaches of opposing teams bid on the yard line from its own end line, with the low bidder winning and starting its offense at the bid. Another proposal would have one team choose the starting yard line and the other team choose whether to cede or take possession at that yard line. We evaluate these proposals in terms of ex post fairness, both in the complete information setting in which teams have information about the chance of winning when starting at different yard lines as common knowledge and in incomplete information setting in which the information about the odds of winning associated starting at various yards is imperfect and dispersed between the two teams.

We find below that various recent proposals yield the same outcome that is ex post fair and envy free in the complete information setting but that auctions provide a more ex post fair outcome than the divide-and-choose procedure when their information is incomplete.

\section{Primitives}

Suppose teams 1 and 2 tie in regulation, turning the game into an overtime. The continuation outcome at the beginning of overtime is characterized by the probability that team 1 would

\footnotetext{
${ }^{1}$ When a college football game goes to overtime, each team is given one possession from its opponent's twenty-five yard line. The leader after those possessions, if there is one, is declared the winner. If the teams remain tied, this continues, switching the order of possessions for each overtime, until one team leads the other at the end of the overtime.

${ }^{2}$ Of the first 355 Nation Football League overtime games 100 were decided by the team winning the coin toss and then winning the game on its first possession (New York Times, 2003c). In 2002 of 25 overtime games, 10 were decided on the first possession (New York Times, 2003b) with 17 games eventually won by the team winning the coin flip.
} 
win. Let $a(x)+\alpha\left(s_{1}, s_{2}\right)$ and $b(y)+\beta\left(s_{1}, s_{2}\right)$ denote team 1's probability of winning when it begins offense at $x$ from its end zone and when team 2 begins its offense at $y$ from its end zone, respectively, where $s_{i}$ is signal team $i=1,2$ observes but its opponent does not. Obviously, $1-b(y)-\beta\left(s_{1}, s_{2}\right)$ and $1-a(x)-\alpha\left(s_{1}, s_{2}\right)$ will then represent team 2's probability of winning when it begins offense at $y$ and when team 1 begins its offense at $x$, respectively. We assume that $s_{i}$ is distributed over $[0,1]$ according to a cdf $F_{i}(\cdot)$ which has a positive density $f_{i}$ and that $\beta(\cdot, \cdot)-\alpha(\cdot, \cdot)$ is nondecreasing; i.e., an increase in the signals make each team prefer ceding a possession to having a possession at a given yard line. The signals thus capture the teams' private assessments about how the overtime game may unfold. Each team (and its coach) maximizes its winning probability.

It is reasonable to assume that $f(\cdot)$ is increasing and $g(\cdot)$ is decreasing; i.e., the further away from its end zone a team begins its offense, the higher is its chance to win. We also assume that $a, b, \alpha, \beta$ are continuous. Finally, we assume that $\forall\left(s_{1}, s_{2}\right), a(0)+\alpha\left(s_{1}, s_{2}\right) \leq b(0)+\beta\left(s_{1}, s_{2}\right)$ and $a(100)+\alpha\left(s_{1}, s_{2}\right) \geq b(100)+\beta\left(s_{1}, s_{2}\right)$. This last assumption means that a team would rather cede possession to the opponent team at its end zone than have a possession at its own end zone. Graphically, it simply means that the curves cross, as illustrated in Figure 1.

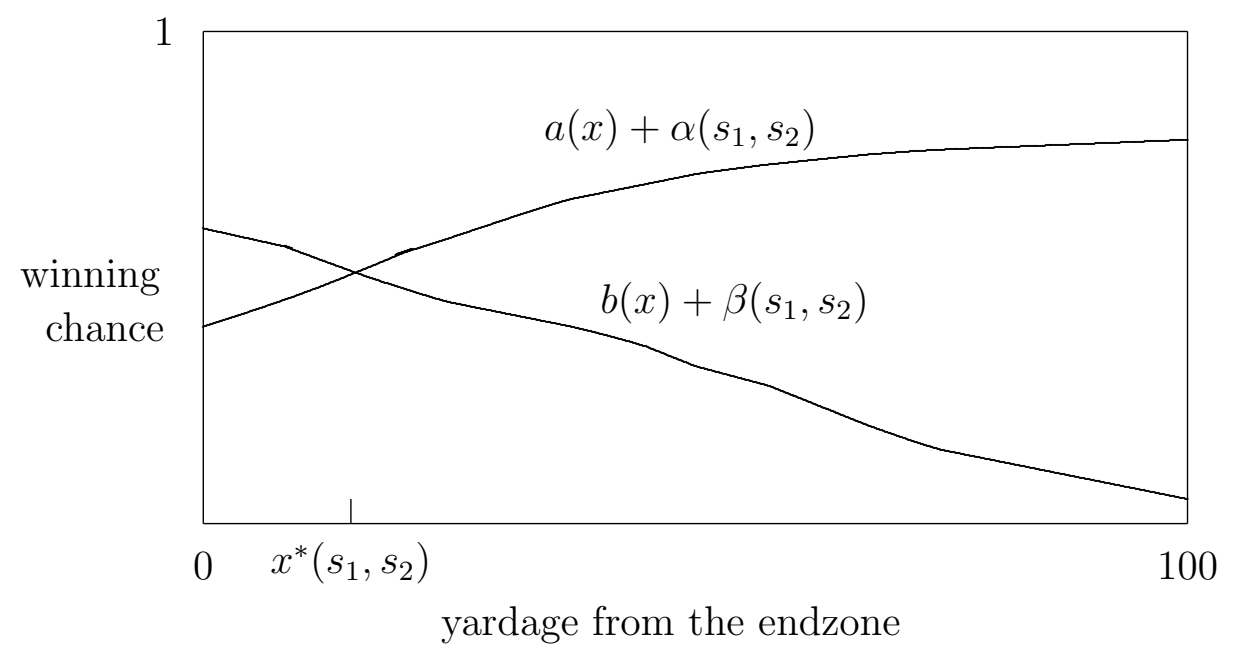

Figure 1

Of special interest is the intersection $x^{*}\left(s_{1}, s_{2}\right)$ of the two curves. This value constitutes an envy-free solution in the sense that both teams are indifferent to a possession at that yardage. In fact, the problem of assigning an overtime possession is isomorphic to the wellknown "cake-cutting" problem, except for the common value element in our problem. ${ }^{3}$ In the

\footnotetext{
${ }^{3}$ In the standard cake cutting problems, each player is assumed to know the exact value of the cake, so the common value problem does not exist. See Steinhaus (1948).
} 
presence of incomplete information, an envy free division may be difficult to implement, nor is it necessarily desirable. We rather focus on the following properties for a fair assignment rule.

EX ANTE SYMMETRY: An assignment rule should treat both teams ex ante symmetrically in the sense that switching the teams in the way the rule applies should not affect the odds of winning for each team ex ante. This property is a minimum requirement of fairness. The current system of flipping coin is ex ante symmetric. So is any anonymous auction rule. The divide-and-choose rule, with a coin flip to determine the divider, is also ex ante symmetric.

Non-ARBITRARINESS: A fair assignment rule should allow the players' abilities and effort - rather than the way the rule assigns the possession - to determine the outcome. In particular, it should minimize the extent to which the fortune of each team is affected by the assignment. We say a rule is non-arbitrary if, for each state $\left(s_{1}, s_{2}\right)$, each team's winning probability at the start of overtime play is non-random under that rule. Formally, a rule $M$ is non-arbitrary if the highest winning probability $\bar{\phi}_{M}\left(s_{1}, s_{2}\right)$ and the lowest winning probability $\underline{\phi}_{M}\left(s_{1}, s_{2}\right)$ it induces for team 1 at the start of the play coincide for almost every $\left(s_{1}, s_{2}\right)$. This feature is precisely what is missing in the current system: The winning probability at the start of an overtime play is random, depending on the realized outcome of the coin flip. The proposed rules may or may not be non-arbitrary depending on the equilibrium play of the assignment games the rules induce.

Non-arbitrariness is certainly desirable. But it is unclear how to compare a non-arbitrary rule with an arbitrary rule, if they implement different winning probabilities on average. The desirability of the former will be clear-cut, however, if the winning probabilities implemented by the two rules are in the same range. We say ex ante symmetric and non-arbitrary rule $A$ is fairer than an ex ante symmetric rule $B$ if

$$
\mathbb{E}_{\left(s_{1}, s_{2}\right)}\left[\underline{\phi}_{B}\left(s_{1}, s_{2}\right)\right]<\mathbb{E}_{\left(s_{1}, s_{2}\right)}\left[\phi_{A}\left(s_{1}, s_{2}\right)\right]<\mathbb{E}_{\left(s_{1}, s_{2}\right)}\left[\bar{\phi}_{B}\left(s_{1}, s_{2}\right)\right]
$$

We are ready to analyze different rules. Below we consider two different types of rules: (a) $\gamma$-auction: Both teams bid simultaneously on the yardage, the lower bidder wins and has possession at $\gamma$. low bid $+(1-\gamma)$. high bid, for some $\gamma \in[0,1]$; (b) Divide-and-choose: A coin is flipped to choose a team; the team proposes a yardage; and the other team chooses between having a possession at that yard and giving a possession to other team at that yardage (from its own end line). 


\section{Benchmark: Complete Information}

Suppose $\alpha\left(s_{1}, s_{2}\right)=\beta\left(s_{1}, s_{2}\right) \equiv 0$. In this case, the teams have completely common opinion about what the odds of winning will be for each team having possession at any given yard. Although this is very unrealistic, the complete information case delivers a very clean analysis and illustrates some desirable features of the recently proposed rules, so it can serve as a useful benchmark. We first characterize the equilibrium of the two proposed rules $4^{4}$

Proposition 1 In any $\gamma$-auction rule with $\gamma \in[0,1]$, it is an equilibrium for each team to bid $x^{*}$ such that $f\left(x^{*}\right)=g\left(x^{*}\right)$. The divide-and-choose rule has an equilibrium in which a team chosen (at random) to pick a yard proposes $x^{*}$. Team 1 wins with probability $f\left(x^{*}\right)$ in any equilibrium.

Proof. Consider first a $\gamma$-auction, $\gamma \in[0,1]$. Suppose team 2 bids $x^{*}$. Team 1's payoff from bidding any $x$ is

$$
\pi_{1}\left(x, x^{*}\right)= \begin{cases}a\left(\gamma x+(1-\gamma) x^{*}\right) & \text { if } x<x^{*} \\ \frac{a\left(x^{*}\right)+b\left(x^{*}\right)}{2} & \text { if } x=x^{*} \\ b\left(\gamma x^{*}+(1-\gamma) x\right) & \text { if } x>x^{*}\end{cases}
$$

Since $\frac{a\left(x^{*}\right)+b\left(x^{*}\right)}{2}=b\left(x^{*}\right)=a\left(x^{*}\right), \pi_{1}\left(x, x^{*}\right)$ is nondecreasing $x$ for any $x \leq x^{*}$ and nonincreasing in $x$ for $x>x^{*}$. So, the payoff is maximized at $x=x^{*}$. Hence, there is no profitable deviation for coach 1 . A symmetric argument works for team 2 , using the fact that $1-a\left(x^{*}\right)=1-b\left(x^{*}\right)$.

Now consider the divide-and-choose rule. Suppose without loss team 1 is chosen to propose a starting yard line and team 2 decides on possession. If team 1 proposes $x$, team 2 will choose $\min \{1-b(x), 1-a(x)\}$, making team 1's payoff equal $\min \{a(x), b(x)\}$, graphed by the lower envelope of the two curves. Clearly, the payoff is maximized at $x^{*}$.

The uniqueness in the probability of winning follows from the fact that each game is a two-person zero sum game. I

It follows that these rules are non-arbitrary. Under very weak conditions these rules are fairer than the current rule. In the current rule, the starting yard line for a team with a possession is a random variable resulting from the opponent's kickoff. It is a reasonable to assume

Assumption A0: In the current system, a team that wins a coin toss chooses an offense; and there exists $x_{0} \in X \cap Y, x_{0} \neq x^{*}$, where $X$ are $Y$ are respectively the support of team 1 and team 2's starting position from one's own end line that results from receiving a kickoff.

\footnotetext{
${ }^{4}$ Part of the analysis here was developed independently by Che (2003) and Kraska (2004).
} 
This assumption is reasonable; in particular the second part simply assumes there to be some common starting position, differing from $x^{*}$, that arises with positive density from receiving a kickoff.

Corollary 1 Given Assumption A0, a $\gamma$-auction and the divide-and-choose rule are fairer than the current system.

Proof. Let $\phi_{M}$ is the probability of winning at the start of overtime play under $M=0, \gamma, d$ representing respectively the current system, a $\gamma$-auction, and the divide-and-choose rule. Let $x_{0} \in X \cap Y, x_{0} \neq x^{*}$. Then,

$$
\underline{\phi}_{0} \leq \min \left\{a\left(x_{0}\right), b\left(x_{0}\right)\right\}<a\left(x^{*}\right)=\phi_{\gamma}=\phi_{d}<\max \left\{a\left(x_{0}\right), b\left(x_{0}\right)\right\} \leq \bar{\phi}_{0}
$$

Since the two rules are non-arbitrary, the proof is complete.

That these two rules implement the envy-free solution has an additional feature: $A$ better team has a higher chance to win. To see this, say team 1 is more capable than team 2 if $a(x) \geq 1-b(x)$ for all $x$, and strictly so if the inequality holds strictly. That is, team 1 is more likely to win than team 2 , if starting its offense at the same position or when facing an offense by its opponent team at the same yardage. Likewise, two teams are equally capable if $a(x)=1-b(x)$ for all $x$.

Corollary 2 In each of the $\gamma$-auction and divide-and-choose rule, a (strictly) more capable team is (strictly) more likely to win, and equally capable teams have the equal chance of winning at the start of an overtime play in equilibrium.

Proof. Let $x^{*}$ be an equilibrium bid. Without any loss, suppose team 1 is more capable than team 2. Then, $a\left(x^{*}\right) \geq 1-b\left(x^{*}\right)$. By Proposition 1, $a\left(x^{*}\right)=b\left(x^{*}\right)$. Combining the two facts, we have $a\left(x^{*}\right) \geq \frac{1}{2}$. The inequality is strict if team 1 is strictly more capable than team 2 , and becomes an equality if two teams are equally capable. I

Under the current system, two equally capable teams will not have the equal chance of winning after the assignment, except by a mere coincidence. Clearly, the equally capable teams have the same chance of winning prior to coin tossing, but what matters is the winning chance after the coin tossing, which is not necessarily a half even when the teams are equally capable. 


\section{Incomplete Information}

We now consider the general case where $\alpha$ and $\beta$ need not vanish. We first characterize the equilibrium of the $\gamma$-auction.

Proposition 2 Any equilibrium of $\gamma$-auction is a pure strategy profile $\left(x^{\gamma}\left(s_{1}\right), y^{\gamma}\left(s_{2}\right)\right)$ that are strictly increasing.

Proof. We first consider a $\gamma$-auction, and fix an equilibrium (possibly in mixed strategies). A standard argument shows that the support of the teams' equilibrium bids coincide and have no gaps and no mass points. Suppose team 2's equilibrium bid is distributed according to a cdf $G(y)$, and let $H\left(s_{2} \mid y\right)$ be the conditional distribution of type $s_{2}$ making bid $y$ (i.e., $\left.F_{2}\left(s_{2}\right)=\int_{y} H\left(s_{2} \mid y\right) d G(y)\right)$. Since $y$ has no mass point, team 1 with type $s_{1}$ receives payoff from bidding $x$ in the equilibrium support:

$$
\begin{gathered}
\Pi_{1}^{\gamma}\left(x ; s_{1}\right):=\int_{y \geq x} \int_{s_{2}}\left[a(\gamma x+(1-\gamma) y)+\alpha\left(s_{1}, s_{2}\right)\right] d H\left(s_{2} \mid y\right) d G(y) \\
\quad+\int_{y<x} \int_{s_{2}}\left[b((1-\gamma) x+\gamma y)+\beta\left(s_{1}, s_{2}\right)\right] d H\left(s_{2} \mid y\right) d G(y) .
\end{gathered}
$$

Note that

$$
\frac{\partial^{2} \Pi_{1}^{\gamma}\left(x ; s_{1}\right)}{\partial x \partial s_{1}}=\int_{s_{2}}\left[\frac{\partial\left(\beta\left(s_{1}, s_{2}\right)-\alpha\left(s_{1}, s_{2}\right)\right)}{\partial s_{1}}\right] d H\left(s_{2} \mid x\right)>0 .
$$

This means that the best response correspondence is monotonic (i.e., every selection is nondecreasing). It follows that the best response is a singleton for almost every $s_{1}$. Since the same holds for team 2, we conclude that the equilibrium is in pure strategies. No mass point implies also that the equilibrium strategies must be strictly increasing.

An immediate consequence of Proposition 2 is that a $\gamma$-auction is non-arbitrary. Again, a $\gamma$-auction is fairer than the current rule under a weak condition. Modify Assumption A0 by replacing $X$ and $Y$ with state specific $X\left(s_{1}, s_{2}\right)$ and $Y\left(s_{1}, s_{2}\right)$, and call it Assumption A0'.

Corollary 3 Given Assumption A0', a $\gamma$-auction is fairer than the current rule.

Proof. Let $x_{0} \in X\left(s_{1}, s_{2}\right) \cap Y\left(s_{1}, s_{2}\right), \forall\left(s_{1}, s_{2}\right)$. Let $\left(x^{\gamma}(\cdot), y^{\gamma}(\cdot)\right)$ be an equilibrium of the $\gamma$-auction game. Since team 1 has an option of simply bidding $x_{0}$ in a $\gamma$-auction, $\forall\left(s_{1}, s_{2}\right)$,

$$
\begin{aligned}
\phi_{\gamma}\left(s_{1}, s_{2}\right) \geq\left(a\left(\gamma x_{0}+(1-\gamma) y^{\gamma}\left(s_{2}\right)\right)+\alpha\left(s_{1}, s_{2}\right)\right) \cdot 1_{\left\{x_{0} \leq y^{\gamma}\left(s_{2}\right)\right\}} \\
+\left(b\left(\gamma y^{\gamma}\left(s_{2}\right)+(1-\gamma) x_{0}\right)+\beta\left(s_{1}, s_{2}\right)\right) \cdot 1_{\left\{x_{0}>y^{\gamma}\left(s_{2}\right)\right\}} \\
\geq \min \left\{a\left(x_{0}\right)+\alpha\left(s_{1}, s_{2}\right), b\left(x_{0}\right)+\beta\left(s_{1}, s_{2}\right)\right\} \geq \underline{\phi}_{0}\left(s_{1}, s_{2}\right) .
\end{aligned}
$$


The first inequality holds since $x_{0}$ need not be a best response and the second follows since $a(\cdot)$ is increasing and $b(\cdot)$ is decreasing. Since $\left(x^{\gamma}, y^{\gamma}\right)$ is increasing, the inequalities must be strict for a positive measure of $\left(s_{1}, s_{2}\right)$.

A symmetric argument for team 2 and the fact that the game is zero sum give

$$
\phi_{\gamma}\left(s_{1}, s_{2}\right) \leq \bar{\phi}_{0}\left(s_{1}, s_{2}\right), \forall\left(s_{1}, s_{2}\right)
$$

again with the inequalities being strict for a positive measure of $\left(s_{1}, s_{2}\right)$. It thus follows that

$$
\mathbb{E}_{\left(s_{1}, s_{2}\right)}\left[\underline{\phi}_{0}\left(s_{1}, s_{2}\right)\right]<\mathbb{E}_{\left(s_{1}, s_{2}\right)}\left[\phi_{\gamma}\left(s_{1}, s_{2}\right)\right]<\mathbb{E}_{\left(s_{1}, s_{2}\right)}\left[\bar{\phi}_{0}\left(s_{1}, s_{2}\right)\right]
$$

Since the $\gamma$-auction is non-arbitrary, the proof is complete.

What about the divide-and-choose rule? For this rule to be ex ante symmetric, the divider and the chooser must be selected by a coin flip. Hence, even though a pure strategy equilibrium may exist for a realized coin flip, the probability of winning at the start of the overtime need not be non-random. For this reason, in general the divide-and-choose rule is not non-arbitrary. In fact, we can present a case favoring an auction over the divide-and-choose rule.

Proposition 3 In a divide-and-choose rule, the chooser is better off on average than the divider. A $\gamma$-auction is fairer than a divide-and-choose rule (with a coin flip).

Proof. Consider the divide-and-choose rule. Suppose a coin flip picks team 1 to be the divider. Suppose in the ensuing equilibrium, the yard line proposed by team 1 is distributed over support $X_{d}$ according to $K(x)$. For each $x \in X_{d}$, let $L\left(s_{1} \mid x\right)$ be the cdf of team 1's type proposing $x$. (Hence, $F_{1}\left(s_{1}\right)=\int_{x} d L\left(s_{1} \mid x\right) d K(x)$.) Let $\phi_{d}^{-}\left(s_{1}, s_{2}\right)$ denote the team 1's equilibrium payoff (i.e., winning chance) in state $\left(s_{1}, s_{2}\right)$ when it is the divider. Likewise, we use $\phi_{d}^{+}\left(s_{1}, s_{2}\right)$ to denote team 1's equilibrium payoff in state $\left(s_{1}, s_{2}\right)$ when it is the chooser.

In equilibrium, observing $x \in X_{d}$ must lead team 2 with any type say $s_{2}$ to infer correctly about the the conditional distribution of team 1's type making that proposal, and chooses a better option. Consequently, team 1's equilibrium payoff condition on proposing $x$ is

$$
\min \left\{\int_{s_{1}}\left(a(x)+\alpha\left(s_{1}, s_{2}\right)\right) d L\left(s_{1} \mid x\right), \int_{s_{1}}\left(b(x)+\beta\left(s_{1}, s_{2}\right)\right) d L\left(s_{1} \mid x\right)\right\} .
$$

Hence, for that $s_{2}$,

$$
=\int_{x \in X_{d}}^{\mathbb{E}_{s_{1}}\left[\phi_{d}^{-}\left(s_{1}, s_{2}\right)\right]} \min \left\{\int_{s_{1}}\left(a(x)+\alpha\left(s_{1}, s_{2}\right)\right) d L\left(s_{1} \mid x\right), \int_{s_{1}}\left(b(x)+\beta\left(s_{1}, s_{2}\right)\right) d L\left(s_{1} \mid x\right)\right\} d K(x) .
$$


Now consider $\gamma$-auction characterized by a pure strategy equilibrium $\left(x^{\gamma}(\cdot), y^{\gamma}(\cdot)\right)$. In that auction equilibrium, team 1 has an option of bidding precisely the yard line it would have proposed in an equilibrium of the divide-and-choose rule when it is the divider. Hence, for each $s_{2}$,

$$
\begin{aligned}
\mathbb{E}_{s_{1}}\left[\phi_{\gamma}\left(s_{1}, s_{2}\right)\right] \geq \int_{x \in X_{c}} & {\left[\left\{\int_{s_{1}}\left(a\left(\gamma x+(1-\gamma) y^{\gamma}\left(s_{2}\right)\right)+\alpha\left(s_{1}, s_{2}\right)\right) d L\left(s_{1} \mid x\right)\right\} \cdot 1_{\left\{x \leq y^{\gamma}\left(s_{2}\right)\right\}}\right.} \\
+ & \left.\left\{\int_{s_{1}}\left(b\left(\gamma y^{\gamma}\left(s_{2}\right)+(1-\gamma) x\right)+\beta\left(s_{1}, s_{2}\right)\right) d L\left(s_{1} \mid x\right)\right\} \cdot 1_{\left\{x>y^{\gamma}\left(s_{2}\right)\right\}}\right] d K(x) \\
\geq & \int_{x \in X_{c}} \min \left\{\int_{s_{1}}\left(a(x)+\alpha\left(s_{1}, s_{2}\right)\right) d L\left(s_{1} \mid x\right), \int_{s_{1}}\left(b(x)+\beta\left(s_{1}, s_{2}\right)\right) d L\left(s_{1} \mid x\right)\right\} d K(x) \\
= & \mathbb{E}_{s_{1}}\left[\phi_{c}^{-}\left(s_{1}, s_{2}\right)\right] \geq \mathbb{E}_{s_{1}}\left[\underline{\phi}_{c}\left(s_{1}, s_{2}\right)\right],
\end{aligned}
$$

where the first inequality follows from the fact that mimicking the strategy of the divider is not necessarily an equilibrium strategy in the auction game, the second follows from the fact that $a(\cdot)$ is increasing and $b(\cdot)$ is decreasing, and the last equality follows from (1). Again given the monotonicity of $\left(x^{\gamma}, y^{\gamma}\right)$, the inequalities must be strict for a positive measure of $s_{2}$.

It follows that

$$
\mathbb{E}_{\left(s_{1}, s_{2}\right)}\left[\phi_{\gamma}\left(s_{1}, s_{2}\right)\right]>\mathbb{E}_{\left(s_{1}, s_{2}\right)}\left[\phi_{c}^{-}\left(s_{1}, s_{2}\right)\right] \geq \mathbb{E}_{\left(s_{1}, s_{2}\right)}\left[\underline{\phi}_{c}\left(s_{1}, s_{2}\right)\right]
$$

A symmetric argument for team 2 when it is a divider and the fact that the game is zero sum give

$$
\mathbb{E}_{\left(s_{1}, s_{2}\right)}\left[\phi_{\gamma}\left(s_{1}, s_{2}\right)\right]<\mathbb{E}_{\left(s_{1}, s_{2}\right)}\left[\phi_{c}^{+}\left(s_{1}, s_{2}\right)\right] \leq \mathbb{E}_{\left(s_{1}, s_{2}\right)}\left[\bar{\phi}_{c}\left(s_{1}, s_{2}\right)\right]
$$

The string of inequalities proves both statements (recalling that a $\gamma$-auction is non-arbitrary).

Although some favors the divide-and-choose rule (see Kraska (2004)), the appeal of this rule does not extend to the more realistic incomplete information setting. The divide-andchoose procedure does not entail an envy-free division in that setting since the chooser's choice will likely cause the divider to regret her division ex post. As Proposition 3 confirms, the rule is unfair to the divider, for she is likely to be taken advantage of by the chooser. Coin tossing to determine the roles will restore ex ante fairness, but will not fix the ex post fairness, and thus will likely have the same unappeal as the current system, albeit in a diminished scale. As the proposition suggests, auctions appear to provide a more fairer and thus desirable outcome. 


\section{Conclusion}

When the Wall Street Journal ran an online forum on this issue (Wall Street Journal, 2003), Tom Donahoe, then president and general manager of the Buffalo Bills, wrote "[w]e don't like the current system. It just seems that too much depends on the coin flip - who wins it and who loses it." Donahoe wrote of the auction proposal being "way too revolutionary for the National Football League. It sounds more like a videogame to me. I couldn't see that happening." However, the NFL's steady increase of coaching decisions to enhance fairness, e.g., the challenge rule for instant replay reviews of plays, are similar in spirit.

Although the current paper has framed its analysis in the context of the NFL overtime, the implications of our analysis goes much beyond that setting. The issue of fair division arises in many real world contexts ranging from dispute resolution, resource allocation to international trade negotiation. While the extant research on fair division assumes that players have full information about the objects being divided, this condition is hardly realistic in many relevant situations. The current paper shows that accounting for the more realistic common value features changes the performances of alternative division procedures that are known to produce the same (i.e., envy-free) outcome. Our analysis suggests that, with two players, auctions tend to provide an ex post fairer outcome than the much heralded divide-and-choose procedure in such a setting.

\section{References}

[1] Che, Y.-K., 2003, "Auctioning off the Right to Start an Offense in an NFL Overtime," Correspondence to Don Hausch, October 14.

[2] Kraska, W., 2004, "Analysis Of Proposed Overtime Auctions," May 1, http://www.footballcommentary.com/otauctions.htm

[3] New York Times, 2003a, N.F.L. Ponders Changing How Overtime Is Played, January 25, Section D, page 5 .

[4] New York Times, 2003b, N.F.L. Decides Not to Make Changes, for Now, March 27, Section S, page 5 .

[5] New York Times, 2003c, Heads or Tails Is No Way To Determine Overtime, November 4, Section D, page 1.

[6] Steinhaus, H., 1948, "The Problem of Fair Division," Econometrica, 16, 101-104. 
[7] Wall Street Journal Online, 2003, Should the Outcome of a Coin Flip Mean So Much in NFL Overtime?, December 22. 